\title{
INNOVACIÓN DE PROCESOS EN LA PEQUEÑA Y MEDIANA INDUSTRIA EN EL SECTOR MANUFACTURERO DE LA CIUDAD DE LATACUNGA
}

\section{INNOVATION OF PROCESSES IN THE SMALL AND MEDIUM-SIZED INDUSTRY IN THE MANUFACTURING SECTOR OF THE CITY OF LATACUNGA}

\author{
Cristian Xavier Espín Beltrán \\ Universidad Técnica de Cotopaxi Ingeniería \\ Industrial. cristian.espin@utc.edu.ec.

\section{Cristian Iván Eugenio Pilliza} \\ Universidad Técnica de Cotopaxi Ingeniería \\ Industrial cristian.eugenio@utc.edu.ec

\section{Luisa Carolina Villa Andrade} \\ Universidad Técnica de Cotopaxi Ingeniería \\ Industrial luisa.villa@utc.edu.ec
}

\section{RESUMEN}

Esta comunicación presenta generalidades de la investigación orientada a determinar la innovación de procesos en las micro, pequeñas y medianas industrias en el sector manufacturero de la ciudad de Latacunga, a fin de brindarles apoyo técnico a través de Proyectos formativos, generativos, de titulación, integradores, así como de prácticas pre profesionales. Por medio de un Modelo Manual de Oslo se aplicó una encuesta de información que ayudó a medir la innovación de procesos en cuanto a: Investigación para desarrollar nuevos procesos, realización de actividades de reingeniería, capacitación al personal para la mejora de procesos, 
incorporación de herramientas, máquinas y/o equipos, incorporación de software, adquisición de bienes vinculados a la mejora de procesos, contratación de asistencia técnica o servicios científicos, incorporación de nuevas tecnologías como uso de patentes, marcas, diseños; aplicación de métodos de comercialización para los productos e Innovación de procesos nuevos o significativamente mejorados.

PALABRAS CLAVE: Procesos, innovación, capacitación y tecnología.

\section{ABSTRACT}

This research project focuses on the study of innovation processes in the micro, small and medium industry in the manufacturing sector of the city of Latacunga, has as its main objective innovation in the micro, small and medium industries. Through an Oslo Manual Model, an information survey was applied that helped to measure the innovation of the processes in terms of: Research to develop new processes, Realization of reengineering activities, Training of personnel for the improvement of processes, Incorporation of tools, machines and / or equipment, incorporation of software, acquisition of goods linked to the improvement of processes, contracting of technical assistance or scientific services, incorporation of new technologies such as the use of patents, trademarks, designs; Application of marketing methods for products and Process innovation to improve or improve. It is about providing technical assistance to the micro, small and medium industries of the city's manufacturing sector through Training Projects, Generative Projects, Titling Projects, Integrating Projects and Pre-Professional Practices.

KEY WORDS: Processes, innovation, training and technology.

\section{INTRODUCCIÓN}

La globalización y el desarrollo industrial hace que cada vez los productos tengan que ser innovados continuamente para mantenerse en el mercado y poder ser competitivos. El propósito de este estudio tiene es conocer la innovación de procesos en las micro, pequeñas y medianas industrias de la ciudad de Latacunga, provincia de Cotopaxi, debido a que son nuevas en el mercado y desconocen acerca de la innovación que pueden implementar en su industria o a su vez la situación económica les impide realizarla. A pesar que las micro, pequeñas y medianas industrias de la ciudad de Latacunga son fundamentales en la economía de la provincia y de la ciudad muchas de estas solo se basan en cumplir uno o varios procesos para elaborar el producto sin tener noción de los beneficios que trae el innovarlos.

Por esta razón las micro, pequeñas y medianas industrias de la ciudad de Latacunga deben estar en un constante desarrollo e innovación en sus procesos y así poder sobresalir en el mercado con sus productos y de esta manera obtener ingresos económicos. El sector industrial manufacturero en la ciudad de Latacunga presenta ciertas deficiencias tanto en el manejo de sus recursos económicos y financieros como en la implementación de nuevos procesos productivos, nueva organización institucional, nueva distribución de planta, acceso a nueva tecnología, falta de capacitación al personal.

Esto lleva a plantearnos como objetivo principal el estudio de innovación en procesos en las micro, pequeñas y medianas Industrias en el sector Manufacturero de la ciudad de Latacunga con el fin de conocer y de informar a las Instituciones participantes que la innovación debe ser constante y puedan brindar un mejor servicio y crear productos de mejor calidad, innovando sus procesos y aplicando la mejora continua.

\section{METODOLOGÍA}

Las actividades de Innovación Innovar implica "combinar diferentes tipos de conocimiento, 
competencias, capacidades y recursos" (Fagerberg, 2003) en procura de una ventaja competitiva, sea por disminución en los costos de producción o por el desarrollo de nuevos productos o por cambios en los existentes. Esta combinación, lejos de ser pasiva, implica la realización de esfuerzos explícitos tendientes a mejorar o crear competencias y capacidades tecnológicas (Lall, 2001).

Esos esfuerzos son denominados habitualmente Actividades de Innovación, las cuales consisten, en definitiva, en la ejecución de "actividades científicas, tecnológicas, organizacionales, financieras y comerciales con la intención de implementar un nuevo o significativamente mejorado producto o proceso, un nuevo método de marketing, un nuevo método organizacional en las prácticas de negocios, en la organización del lugar de trabajo o en las relaciones externas" (MO). Las firmas que realizan este tipo de actividades (esfuerzos innovativos) son consideradas innovativas mientras que se denomina innovadoras a las que logran alcanzar resultados (es decir, introducir innovaciones).
Las Actividades de Innovación involucran tanto los esfuerzos en procura de generar nuevos conocimientos como los de adquirir, adaptar o desarrollar conocimientos existentes así como, en general, a las diversas formas de incrementar las capacidades productivas y tecnológicas de la empresa ya sea en su equipamiento como en su dotación de recursos humanos.

\section{Esto incluye:}

- Investigación y Desarrollo (I+D), tanto in house (es decir, al interior de la empresa) o por la vía de un acuerdo o contratación de un agente externo a la firma, que es quien lleva adelante los trabajos;

- Adquisición de tecnología incorporada (equipos, hardware y software) y desincorporada (licencias, patentes);

- Contratación de consultorías y asistencia técnica;

- Actividades de Ingeniería y Diseño Industrial;

- Capacitación del personal.

- Actividades de marketing

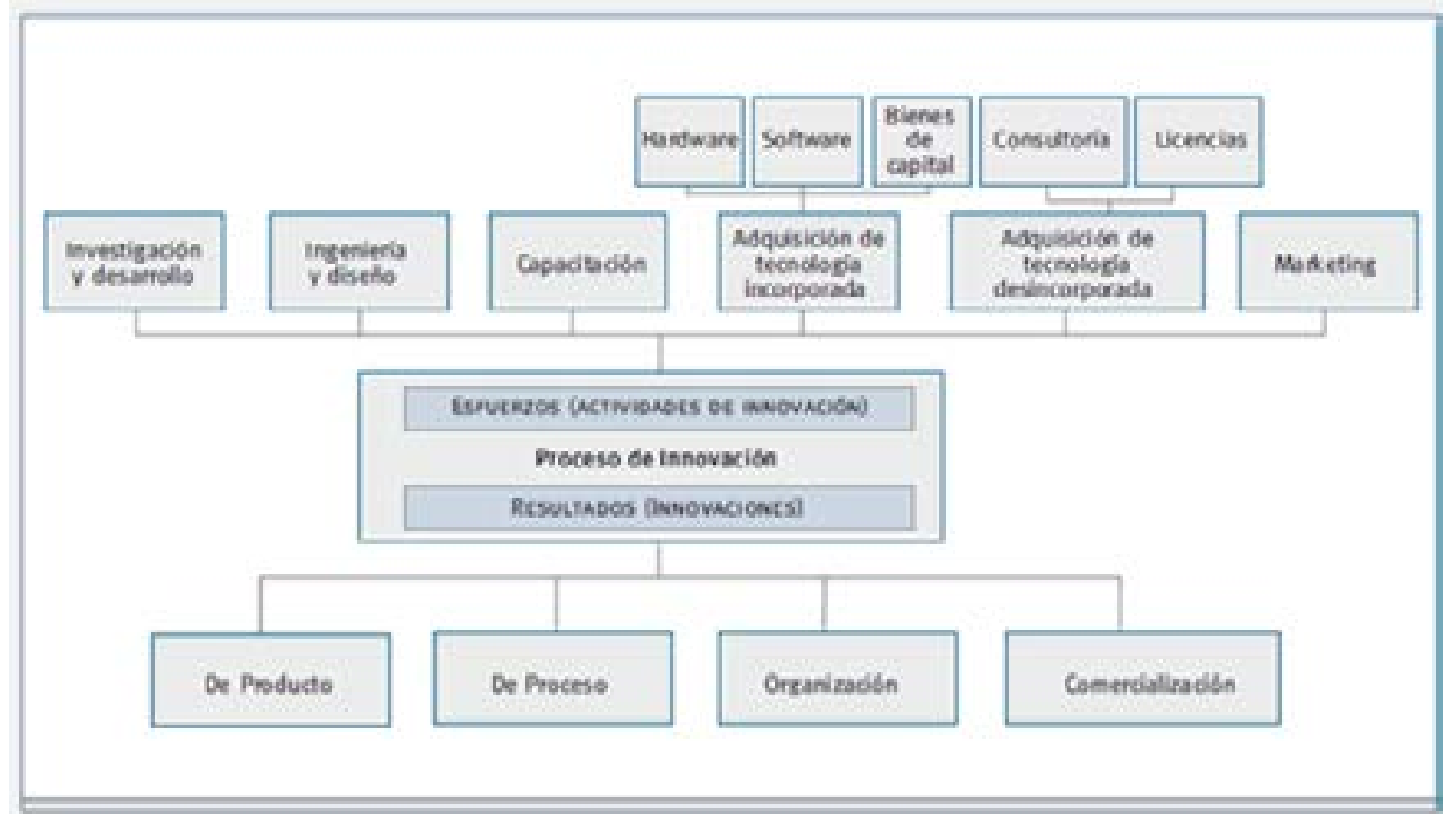

Figura 1. Matriz de Oslo 
Para realizar la identificación de las pequeñas y medianas industrias se recopilará información sobre el número de industrias existentes en el sector objeto de investigación al que pertenecen. Conocer las empresas existentes y lograr los objetivos propuestos en la investigación, se tendrá contacto directo con los representantes de cámara de comercio, cámara de industrias, asociaciones, gremios y artesanos.

Mediante una base de datos emitida por la Cámara de Comercio y Cámara de Industrias de Latacunga, se selecciona las industrias objeto de estudio y se inicia con estrategias de recopilación de datos de las industrias participantes. Se analiza el tipo de innovación implementada (proceso).

Cabe mencionar que al trabajar con el Modelo del Manual de Oslo se debe tener claro que las industrias se clasifican en pequeñas, medianas y grandes industrias, mientras que en nuestro medio las distinguimos como micros, pequeñas, medianas y grandes industrias.

\section{RESULTADOS}

Primero se seleccionó a las pequeñas y medianas industrias del sector manufacturero de la ciudad de Latacunga. Mediante una Base de Datos emitida por la Cámara de Comercio y Cámara de Industrias de Latacunga se pudo conocer las pequeñas y medianas industrias del sector manufacturero de la ciudad de Latacunga. Mediante la socialización del proyecto se pudo seleccionar a las industrias que participarán en el mismo, mediante una hoja de registro se recolectó datos de las personas representantes de cada industria que accedieron a participar de manera voluntaria en el proyecto.

Se aplicó una encuesta de información virtual y física elaborada con el Modelo del Manual de Oslo para medir indicadores de innovación de procesos en las pequeñas y medianas industrias del sector manufacturero de la ciudad de Latacunga.

\section{ANÁLISIS DE LA INFORMACIÓN RECOLECTADA EN LAS ENCUESTAS.}

Tabla 1.Sector al que pertenece la industria.

\begin{tabular}{|l|l|c|c|}
\hline \multicolumn{3}{|c|}{ 1.- ¿SU INDUSTRIA PERTENECE AL SECTOR? } \\
\hline ITEM & RESPUESTA & EMPRESAS & PORCETAJE \\
\hline A & MICRO & 25 & $83 \%$ \\
\hline B & PEQUEÑAS & 2 & $7 \%$ \\
\hline C & MEDIANAS & 3 & $10 \%$ \\
\hline \multicolumn{3}{|l}{} & $100 \%$ \\
\cline { 4 - 4 }
\end{tabular}

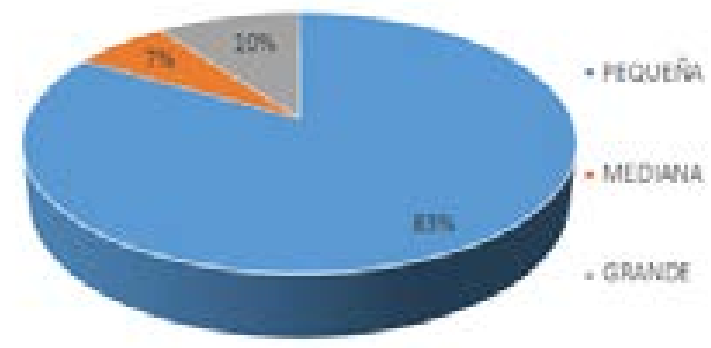

Figura 2. Sector al que pertenece la industria.

Tabla 2. A qué se dedica la industria

\begin{tabular}{|l|l|c|c|}
\hline \multicolumn{4}{|c|}{ 2.- ¿Su Industria se dedica a? } \\
\hline ITEM & RESPUESTA & EMPRESAS & PORCETAJE \\
\hline \multirow{2}{*}{ A } & $\begin{array}{l}\text { PRODUCIR } \\
\text { BIENES }\end{array}$ & 19 & $63 \%$ \\
\hline \multirow{3}{*}{ B } & $\begin{array}{l}\text { PRODUCIR } \\
\text { SERVICIOS }\end{array}$ & 11 & $37 \%$ \\
\hline \multicolumn{3}{|c}{} \\
\cline { 4 - 4 }
\end{tabular}

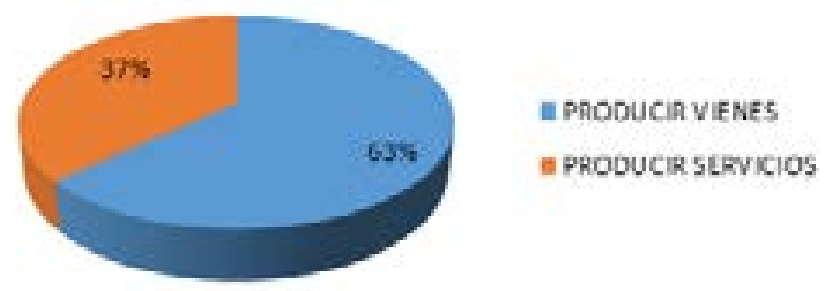

Figura 3. A que se dedica la industria. 


\section{INVESTIGACIÓN Y DESARROLLO}

Tabla 3. Desarrollo de nuevos procesos

\begin{tabular}{|l|l|c|c|}
\hline \multicolumn{3}{|c|}{ 3.- ¿QUÉ TIPO DE INVESTIGACIÓN HA REALIZADO SU INDUSTRIA } \\
PARA DESARROLLAR NUEVOS PROCESOS? \\
\hline ITEM & \multicolumn{1}{|c|}{ RESPUESTA } & INDUSTRIAS & PORCENTAJE \\
\hline A & INVESTIGACIÓN BÁSICA & 13 & $36 \%$ \\
\hline B & INVESTIGACIÓN APLICADA & 6 & $17 \%$ \\
\hline & $\begin{array}{l}\text { DESARROLLO } \\
\text { EXPERIMENTAL }\end{array}$ & 9 & $25 \%$ \\
\hline D & NINGUNA & 8 & $22 \%$ \\
\hline
\end{tabular}
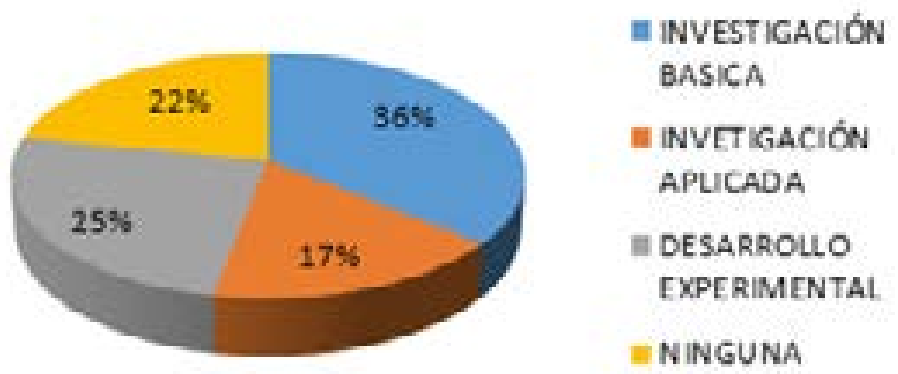

Figura 4. Desarrollo de nuevos procesos Elaborado por: Autores

\section{INGENIERÍA Y DISEÑO}

Tabla 4. Actividades de reingeniería

\begin{tabular}{|l|l|c|c|}
\hline \multicolumn{4}{|c|}{ 4.- ¿SE HA REALIZADO EN SU INDUSTRIA ACTIVIDADES DE } \\
REINGENIERÍA TALES COMO? \\
\hline ITEM & \multicolumn{1}{|c|}{ RESPUESTA } & EMPRESAS & PORCETAJE \\
\hline A & $\begin{array}{l}\text { MODIFICACIÓN AL PROCESO } \\
\text { PRODUCTIVO }\end{array}$ & 9 & $21 \%$ \\
\hline B & $\begin{array}{l}\text { SISTEMA DE ORGANIZACIÓN } \\
\text { DE PRODUCTOS }\end{array}$ & 6 & $14 \%$ \\
\hline C & PRÁCTICAS DE CALIDAD & 11 & $26 \%$ \\
\hline D & $\begin{array}{l}\text { SISTEMAS INTEGRALES DE } \\
\text { PRODUCCIÓN }\end{array}$ & 5 & $12 \%$ \\
\hline E & OTROS & 0 & $0 \%$ \\
\hline F & NINGUNA & 12 & $28 \%$ \\
\hline
\end{tabular}




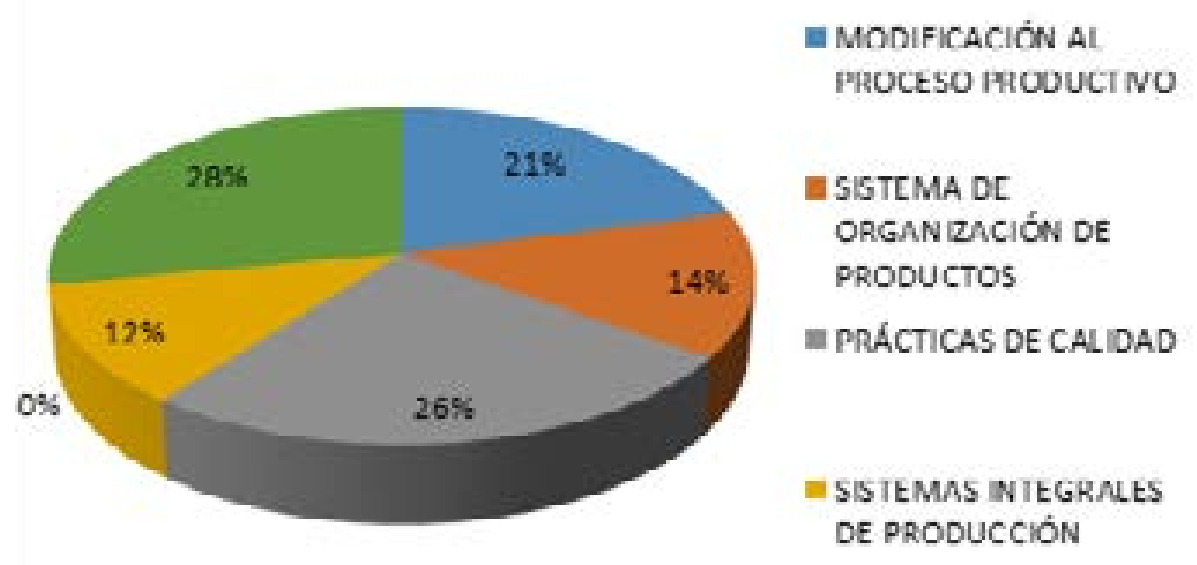

Figura 5. Desarrollo de nuevos productos

\section{CAPACITACIÓN}

Tabla 5. Capacitaciones que mejoren procesos

\begin{tabular}{|c|l|c|c|}
\hline $\begin{array}{l}\text { 5.- ¿SU INDUSTRIA HA BRINDADO CAPACITACIONES QUE PERMITAN } \\
\text { MEJORAR SUS PROCESOS COMO? }\end{array}$ \\
\hline ITEM & \multicolumn{1}{|c|}{ RESPUESTA } & INDUSTRIAS & PORCENTAJE \\
\hline A & $\begin{array}{l}\text { TECNOLOGIAS BLANDAS } \\
\text { (GESTIÓN Y ADMINISTRACIÓN) }\end{array}$ & 3 & $10 \%$ \\
\hline B & $\begin{array}{l}\text { TECNOLOGIAS DURAS } \\
\text { (PROCESOS PRODUCTIVOS) }\end{array}$ & 6 & $20 \%$ \\
\hline C & NINGUNA & 21 & $70 \%$ \\
\hline
\end{tabular}

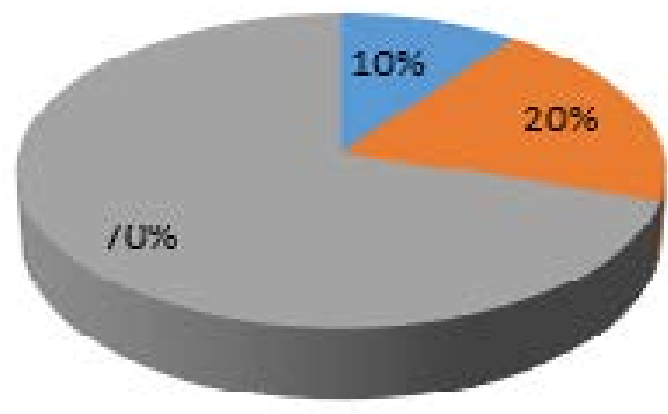

TECNOLOGIAS

BLANDAS (GESTIÓN

Y AUMINISI KACIÚN)

- TECNOLOGIAS

DURAS (PROCESOS

PRODUCTIVOS)

N INGUNA

Figura 6. Capacitaciones que mejoren procesos 
ADQUISICIÓN DE TECNOLOGÍA INCORPORADA (HADWARE, SOFTWARE, BIENES DE CAPITAL)

Tabla 6. Mejora en la industria

\begin{tabular}{|c|c|c|c|}
\hline \multicolumn{4}{|c|}{$\begin{array}{l}\text { 6.- ¿DE LAS SIGUIENTES OPCIONES SEÑALE CUÁL DE ÉSTAS HA MEJORADO } \\
\text { EN SU INDUSTRIA? (TOMAR EN CUENTA QUE REEMPLAZAR NO ES INNOVAR) }\end{array}$} \\
\hline ITEM & RESPUESTA & INDUSTRIAS & PORCENTAJE \\
\hline A & HERRAMIENTAS & 1 & $3 \%$ \\
\hline$B$ & MAQUINARIA & 12 & $36 \%$ \\
\hline C & EQUIPOS & 8 & $24 \%$ \\
\hline $\mathrm{D}$ & NINGUNA & 12 & $36 \%$ \\
\hline
\end{tabular}

$3 \%$

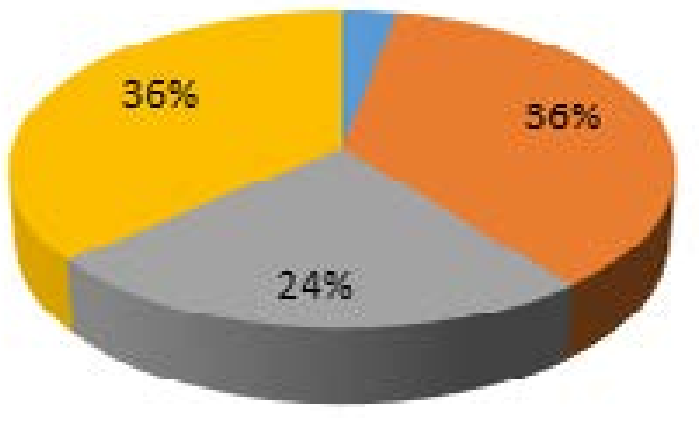

HERRAMIENTAS

MAQUINARIA

- EQU IPOS

NINGUNA

Figura 7. Mejora en la industria

Tabla 7. Software incorporado

7.- ¿QUÉ TIPO DE SOFTWARE HA INCORPORADO SU INDUSTRIA PARA MEJOR SUS PROCESOS?

\begin{tabular}{|c|l|c|c|}
\hline ITEM & \multicolumn{1}{|c|}{ RESPUESTA } & INDUSTRIAS & PORCENTAJE \\
\hline A & $\begin{array}{l}\text { ACTUALIZACIÓN DE SOFTWARE } \\
\text { EXISTENTE EN SU EMPRESA }\end{array}$ & 0 & $0 \%$ \\
\hline B & $\begin{array}{l}\text { IMPLEMENTACIÓN DE NUEVOS } \\
\text { SOFTWARE }\end{array}$ & 3 & $10 \%$ \\
\hline C & NINGUNA & 27 & $90 \%$ \\
\hline \multicolumn{2}{|l}{} & $100 \%$ \\
\hline
\end{tabular}
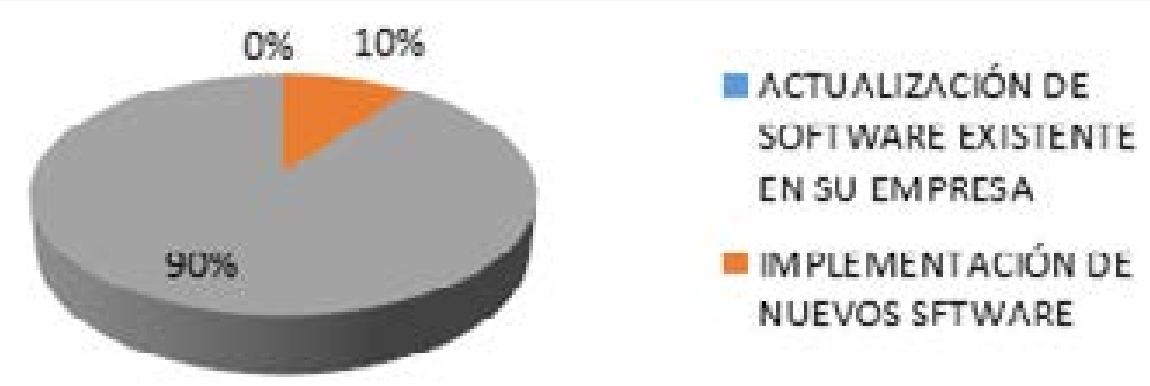

Figura 8. Software incorporado 
Tabla 8. Bienes vinculados a la mejora de procesos

\begin{tabular}{|c|c|c|c|}
\hline \multicolumn{4}{|c|}{ 8.- ¿SU EMPRESA HA ADQUIRIDO NUEVOS BIENES VINCULADOS A } \\
MEJORA SUS PROCESOS? \\
\hline ITEM & RESPUESTA & INDUSTRIAS & PORCENTAJE \\
\hline A & SI & 13 & $43 \%$ \\
\hline B & NO & 17 & $57 \%$ \\
\hline \multicolumn{3}{|c}{} \\
\cline { 3 - 4 }
\end{tabular}

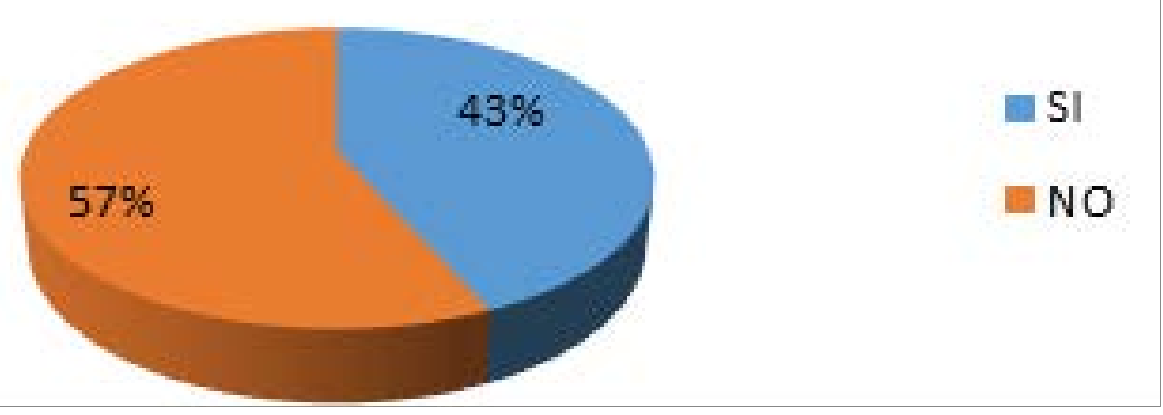

Figura 9. Bienes vinculados a la mejora de procesos

\section{ADQUISICIÓN DE TECNOLOGÍAS DESINCORPORADA (CONSULTORÍAS, LICENCIAS)}

Tabla 9. Asistencia técnica para mejora de procesos

\begin{tabular}{|c|c|c|c|}
\hline \multicolumn{4}{|c|}{$\begin{array}{l}\text { 9.- ¿HA CONTRATADO ASISTENCIA TÉCNICA O SERVICIOS CIENTÍFICOS } \\
\text { PARA MEJORAS LOS PROCESOS DE SU INDUSTRIA? }\end{array}$} \\
\hline ITEM & RESPUESTA & INDUSTRIAS & PORCENTAJE \\
\hline$A$ & $\mathrm{SI}$ & 9 & $30 \%$ \\
\hline$B$ & NO & 21 & $70 \%$ \\
\hline
\end{tabular}

Figura 10. Asistencia técnica para mejora de procesos 
Tabla 10. Incorporación de nuevas tecnologías

\begin{tabular}{|c|l|c|c|}
\hline \multicolumn{4}{|c|}{$\begin{array}{c}\text { 10.- ¿SU INDUSTRIA HA INCORPORADO NUEVAS TECNOLOGÍAS PARA } \\
\text { MEJORAS SUS PROCESOS TALES COMO? }\end{array}$} \\
\hline ITEM & \multicolumn{1}{|c|}{ RESPUESTA } & INDUSTRIAS & PORCENTAJE \\
\hline A & USO DE PATENTES & 5 & $12 \%$ \\
\hline B & INVENTOS NO PATENTADOS & 2 & $5 \%$ \\
\hline C & LICENCIAS & 1 & $2 \%$ \\
\hline D & MARCAS & 5 & $12 \%$ \\
\hline E & DISEÑOS & 6 & $14 \%$ \\
\hline F & KNOW-HOW & 5 & $12 \%$ \\
\hline G & ASISTENCIA TÉCNICA & 8 & $19 \%$ \\
\hline H & SERVICIOS TECNOLÓGICOS & 1 & $2 \%$ \\
\hline I & NINGUNO & 10 & $23 \%$ \\
\hline
\end{tabular}
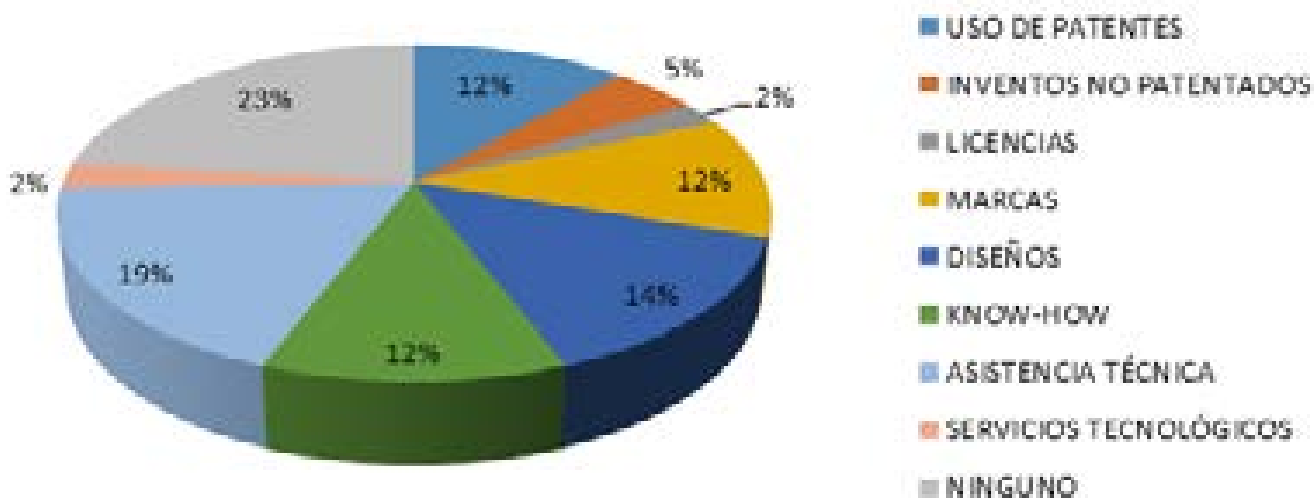

Figura 11. Incorporación de nuevas tecnologías

\section{MARKETING}

Tabla 11. Métodos de comercialización

\begin{tabular}{|c|l|c|c|}
\hline \multicolumn{4}{|c|}{$\begin{array}{l}\text { 11.- ¿HA INCORPORADO NUEVOS MÉTODOS DE COMERCIALIZACIÓN } \\
\text { PARA SUS PROCESOS TALES COMO? }\end{array}$} \\
\hline ITEM & \multicolumn{1}{|c|}{ RESPUESTA } & INDUSTRIAS & PORCENTAJE \\
\hline & $\begin{array}{l}\text { INVESTIGACIÓN DE } \\
\text { MERCADO }\end{array}$ & 5 & $16 \%$ \\
\hline B & TESTEO DE MERCADO & 2 & $6 \%$ \\
\hline & $\begin{array}{l}\text { CAMPAÑAS DE } \\
\text { PUBLICIDAD }\end{array}$ & 11 & $34 \%$ \\
\hline D & NINGUNA & 14 & $44 \%$ \\
\hline
\end{tabular}




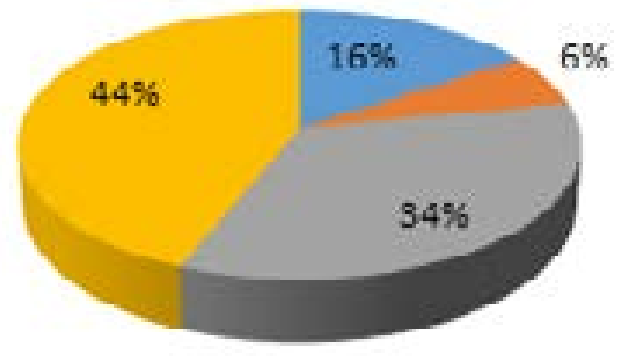

\section{* INVESTIGACIÚN UE MERCADU \\ TTESTFO DF \\ MFRCADO \\ I CAMPAÑAS DF PUBLICIDAD}

NINGUNA

Figura 12. Métodos de comercialización

Tabla 12. Procesos Innovados

\begin{tabular}{|c|l|c|c|}
\hline \multicolumn{3}{|c|}{ 12.- ¿QUÉ PROCESOS HA INNOVADO SU INDUSTRIA? } \\
\hline ITEM & \multicolumn{1}{|c|}{ RESPUESTA } & INDUSTRIAS & PORCENTAJE \\
\hline A & PROCESOS NUEVOS & 3 & $10 \%$ \\
\hline & $\begin{array}{l}\text { PROCESOS } \\
\text { SIGNIFICATIVAMENTE } \\
\text { B }\end{array}$ & & \\
\hline CEJORADOS & NINGUNA & 3 & $10 \%$ \\
\hline \multicolumn{2}{|l|}{} & 24 & $80 \%$ \\
\hline
\end{tabular}

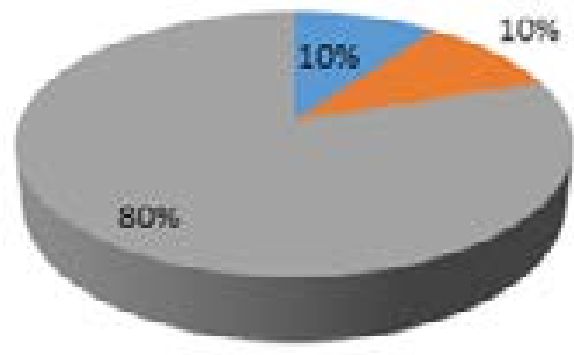

- PROCESOS NUEVOS

PROCESOS

SIGNIFICATNAMENTE

MEJORADOS

ININGUNA

Figura 13. Procesos Innovados

\section{DISCUSIÓN}

La mayoría de empresas que participaron en el estudio fueron microempresas con un $83 \%$ ya que son la que en la zona existen en su mayoría y un $63 \%$ son productores de bienes. En lo que $s$ e refiere a que procesos se ha innovado se obtuvo en un $80 \%$ que no se ha innovado los procesos productivos eso se interpreta que no se incorporado investigación, tecnología, software, capacitación y no ha permitido tener una mejora significativa en los procesos que permitan tener mejores procesos y productos que generen menos costos de producción por ende no son competitivos en el mercado ya que no han sido innovados. 


\section{CONCLUSIONES}

El $80 \%$ de las micro, pequeñas y medianas industrias del sector manufacturero de la ciudad de Latacunga no ha realizado innovación en sus procesos, por lo cual no pueden existir un crecimiento económico y se corre el riesgo de perder mercado ya que sin innovación de procesos no se obtendrá una mejora en el producto y de esa manera no será competencia para las demás industrias.

Según los resultados obtenidos de la encuesta el $28 \%$ de industrias deben implementar alguna clase de reingeniería, tomando en cuenta que son industrias pequeñas que están recién naciendo en el mercado y eso dificulta el conocimiento de la importancia de realizar dichas reingenierías para la mejora de cada industria.

Con el estudio realizado y con un $70 \%$ indicándonos que no se ha brindado capacitación a las industrias sobre innovación de procesos lo que conlleva a disminuir el nivel de competencia entre las industrias.

El $36 \%$ de las industrias carecen de innovación de herramientas, máquinas y equipos por lo cual prefieren reemplazar alguna de éstas sin tener en cuenta que reemplazar no es innovar.

De las industrias participantes son micro, pequeñas y medias industrias en donde el $90 \%$ no a incorporado incorporar algún tipo de software para innovar sus procesos, es por ello que se proporcionará ayuda con los estudiantes de la carrera para que puedan aportar sus conocimientos en las diferentes áreas de las industrias.

El $57 \%$ de Las industrias que no adquieren bienes para la innovación de procesos no generan crecimiento, por lo cual, impartir conocimientos ayuda a analizar y elegir de mejor manera la opción correcta en la adquisición de bienes.
Actualmente el $70 \%$ de las industrias encuestadas no cuentan con asistencia técnica o de algún tipo de servicio que ayude a la innovación de sus procesos, dado que esto genera gastos y algunas industrias al ser nuevas no cuentan con un factor económico, de esta manera los estudiantes de la carrera de Ingeniería industrial serán un recurso humano muy importante al poder brindar apoyo técnico.

Con un $23 \%$ de resultado negativo es posible mencionar que las nuevas tecnologías en las industrias no son incorporadas, muchas de ellas prefieren no realizar ningún tipo de adquisición o incorporación.

El $44 \%$ de industrias encuestadas se puede evidenciar que la mayoría de ellas no realizan esta actividad, demostrando que al no incorporar métodos de comercialización su producto no tiene competencia.

Al concluir la encuesta el $80 \%$ de las industrias al no tener conocimiento de innovación en procesos y asesoramiento adecuando, no obtienen procesos nuevos o procesos significativamente mejorados lo cual generan la inexistencia de mejoras en la producción y por lo tanto en el producto.

\section{LITERATURA CITADA}

Drucker, P. (2000). La disciplina de la innovación. Creatividad e innovación, 157-174.

Escorsa Castells, P., \& Pasola, J. V. (2003). Tecnología e innovación en la empresa (Vol. 148). Univ. Politèc. de Catalunya.

Escorsa Castells, P., \& Pasola, J. V. (2004). Tecnología e innovación en la empresa (Vol. 148). Univ. Politèc. de Catalunya.

Fernández Sánchez, E. (2005). Estrategia de innovación. Editorial Thomson Madrid, España.

Adolfo Acevedo, Carolina Linares. (2008). El proceso de innovación dentro del diseño




estrategico de las organizaciones. Produccion y gestion, 33 .

Horta R, Azua S, Camacho M y Astrigarraga M. (2015). Los procesos de innovacion al interiro de las empresas. Competitividad e innovación en la industria manufacturera en el Uruguay.

Lluis, G. (2017). Objetivoos de la innovacion. Rtribucion de Innovadores.

Transformación de la Matriz Productiva. (2018). Obtenido de Transformación de la Matriz Productiva: http://www.planificacion.gob.ec/ wp-content/uploads/downloads/2013/01/matriz productiva_WEBtodo.pdf

UTC, U. T. (29 de Mayo de 2017). Políticas de Investigación Científica y Desarrollo Tecnológico. Obtenido de http://www.utc.edu. ec/INVESTIGACION/Sistema-de-Investigacion/ lineas-investigacion 